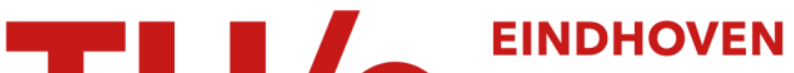

\section{Setting optimal planned leadtimes in configure-to-order assembly systems}

\section{Citation for published version (APA):}

Jansen, S., Atan, Z., Adan, I., \& Kok, T. D. (2019). Setting optimal planned leadtimes in configure-to-order assembly systems. European Journal of Operational Research, 273(2), 585-595. [2].

https://doi.org/10.1016/j.ejor.2018.08.036

DOI:

10.1016/j.ejor.2018.08.036

Document status and date:

Published: 01/03/2019

\section{Document Version:}

Accepted manuscript including changes made at the peer-review stage

\section{Please check the document version of this publication:}

- A submitted manuscript is the version of the article upon submission and before peer-review. There can be important differences between the submitted version and the official published version of record. People interested in the research are advised to contact the author for the final version of the publication, or visit the $\mathrm{DOI}$ to the publisher's website.

- The final author version and the galley proof are versions of the publication after peer review.

- The final published version features the final layout of the paper including the volume, issue and page numbers.

Link to publication

\section{General rights}

Copyright and moral rights for the publications made accessible in the public portal are retained by the authors and/or other copyright owners and it is a condition of accessing publications that users recognise and abide by the legal requirements associated with these rights.

- Users may download and print one copy of any publication from the public portal for the purpose of private study or research.

- You may not further distribute the material or use it for any profit-making activity or commercial gain

- You may freely distribute the URL identifying the publication in the public portal.

If the publication is distributed under the terms of Article $25 \mathrm{fa}$ of the Dutch Copyright Act, indicated by the "Taverne" license above, please follow below link for the End User Agreement:

www.tue.nl/taverne

Take down policy

If you believe that this document breaches copyright please contact us at:

openaccess@tue.nl

providing details and we will investigate your claim. 


\title{
Setting Optimal Planned Leadtimes in Configure-To-Order Assembly Systems
}

\author{
Sjors Jansen*1, Zümbül Atan ${ }^{1}$, Ivo Adan ${ }^{1}$, and Ton de Kok ${ }^{1}$ \\ ${ }^{1}$ Department of Industrial Engineering and Innovation Sciences, Eindhoven University of \\ Technology, Eindhoven, The Netherlands
}

\begin{abstract}
We study a configure-to-order assembly system consisting of multiple parallel subassembly stages and one final assembly stage. Each stage has a stochastic leadtime. The system is controlled by planned leadtimes at each stage. Planned leadtimes are used to plan start and finish times at all stages. The system incurs holding cost for each stage from the planned start time of the stage until the final product is delivered to the customer. In addition, a penalty cost is incurred if the final assembly stage is late. The objective is to set the planned leadtimes for each stage such that the total expected cost is minimized. We prove that the optimal planned leadtimes satisfy a set of Newsvendor equations and show that the probability that a stage is "blamed" for the lateness of the system equals a Newsvendor fractile. We derive structural properties of the optimal solution and compare it with the optimal solution under an alternative holding cost regime.

Keywords: Supply chain management, Assembly systems; Planned leadtimes; Newsvendor equations.
\end{abstract}

\section{Introduction}

Companies that aim to operate with a minimum cost and a high service level face a challenging task of identifying the best operational strategies for achieving these objectives. Attaining these goals is difficult as companies face many uncertainties on the supply, production and demand sides. The magnitudes and effects of these uncertainties on the operations of a company depend on its production strategy. The best strategy can be determined by identifying how deeply the customer order penetrates the company's supply chain (Atan et al. 2017).

A production strategy known as "configure-to-order" strategy is commonly utilized by companies which offer customized and, mostly, expensive products. The end product fulfills the exact customer needs. This is an appealing strategy for high-tech, car manufacturing and white goods industries.

The supply chain of a company using a configure-to-order strategy distinguishes between two main phases. The first phase concerns the orders placed with the suppliers. This phase is forecastdriven and is executed in advance to prevent excessive customer leadtimes. The second phase is

\footnotetext{
${ }^{*}$ Corresponding author: s.w.f.jansen@tue.nl, P.O.Box 513, 5600MB,Eindhoven, The Netherlands
} 
driven by the customer order and concerns the production of the final customer-specific product. The point which divides these two phases is known as customer order decoupling point de Kok \& Fransoo 2003). The focus of this paper is on the second phase of the configure-to-order strategy.

We consider a company that keeps inventory of components only. Production of multiple subassemblies is triggered by a customer order. These subassemblies are then assembled into a final product. Production and assembly leadtimes are random. The subassemblies and final product are customer-specific and thus cannot be kept on stock. Therefore, safety stocks cannot be used as a technique to absorb the uncertainties in the production process. A plan, which sets planned production leadtimes for subassembly and final stages, is used to buffer against uncertainties. We call these production leadtimes planned leadtimes. The planned leadtime is the sum of the average leadtime and a safety time. The difficulty of planning the second phase of the configure-to-order strategy arises from the interactions among multiple processes. A good plan should coordinate the production of subassemblies and ensure on time delivery of the final product.

The company incurs cost for holding each subassembly and the final product. These costs are incurred from the planned start time of a stage (subassembly or final) until the delivery of the final product to the customer. Since each stage adds value to the final product, the total holding costs per time unit increase over time and are maximal at the final assembly stage. A penalty cost is incurred for late delivery of the product to the customer. The objective of this paper is to determine the planned leadtimes for all the stages such that the sum of expected holding and penalty costs is minimized.

Setting planned leadtimes to ensure timely delivery of customer orders is a key tactical decision for many companies (Atan et al. 2016). Multiple researchers have developed models to assist the companies with this decision. The distinguishing feature of our work is the way holding cost are accounted for. All previous work assume that the holding costs are incurred from the actual start times of the stages. This accounting scheme is realistic when materials are supplied exactly when they are needed, for example in a Just In Time (JIT) enviroment. In our paper, instead of incurring holding cost from the actual start time of a stage, we start to incur holding cost of a stage from its planned start time. This is motivated by practice. Companies that rely on safety times instead of safety stocks to protect against uncertainties are mostly the ones that produce capital-intense products. These companies allocate expensive material to production stages. The material is ordered from external suppliers or from other departments within the same company. The material is ready at the planned start times. If production cannot start at the planned start time, the material needs to be stored and interest for invested capital should be paid. These are the cost that we consider as holding cost in this paper. As these cost need to be paid for, regardless of whether production at a stage is started at its planned start time or not, we incur these holding cost from the planned start 
time.

In addition to introducing a new holding cost accounting scheme, we contribute to the literature by introducing the concept of a "blame policy", which can be applied to all planned leadtime optimization problems for any assembly system structure with stochastic leadtimes. For each realization of leadtimes, the blame policy identifies the stage that causes the late delivery of the product. For a system operating according to the optimal planned leadtime solution, we prove that the blame probability of each stage satisfies a Newsvendor equation. This equation states that the probability that a stage is blamed for late delivery is proportional to the value the stage adds to the final product. We end up with a set of Newsvendor equations, which can be numerically solved to obtain the unique optimal solution to the cost minimization problem.

We compare our cost function with the cost function used in Atan et al. (2016). The authors study the same configure-to-order system with the holding costs incurred from the actual start times of the stages. We derive structural results for the cost difference. We prove that our optimal solution allocates more time to each subassembly stage and less time to the final assembly stage compared to the optimal solution to the cost function of Atan et al. (2016). We present a numerical experiment illustrating that if the service level requirement is high, the difference between the optimal costs is marginal. However, the difference between the optimal planned leadtimes is significant. Our solution leads to a significantly higher probability that intermediate deadlines are being met.

Our numerical experiments also unveiled that in some cases the optimal solution to the cost function in Atan et al. (2016) can contain negative planned leadtimes. Negative planned leadtimes are counter-intuitive and can lead to difficulties when implementing in practice. We prove that for our cost function, the optimal planned leadtimes are always non-negative.

The remainder of this paper is organized as follows. In Section 2 we provide a brief review of the literature on setting the planned leadtimes. In Section 3 we introduce notations and formulate the optimization problem. In Section 4 we define the blame policy and derive a set of Newsvendor equations. We describe properties of the cost function and the optimal solution in Section 5 and compare the optimal solution with the one obtained by Atan et al. (2016). Numerical results are presented in Section 6. We provide concluding remarks and discuss future research directions in Section 7.

\section{Literature Review}

Most of the earlier work on setting planned leadtimes focuses on single-stage systems and systems with specific structures. For a single-stage system, Weeks (1981) shows the equivalence of the planned leadtime problem to the well-known Newsvendor problem. Subsequently, Matsuura \& Tsubone 
(1993), Matsuura et al. (1996) and Buzacott \& Shanthikumar (1994) study the single-stage problem and conclude that safety times should be preferred over safety stock if the company has accurate forecasts on the shipments over the leadtime.

Multi-stage systems are more difficult to analyze as optimal decisions across stages are not independent. Earlier work on serial multi-stage systems includes Yano (1987a) and Gong et al. (1996). Yano (1987a) develops an algorithm to solve the problem of determining the optimal planned leadtimes for serial systems and Gong et al. (1996) proves that this problem is equivalent to the problem of determining the optimal base-stock levels in serial inventory systems. Elhafsi (2002) also study a serial production system with the objective to determine the optimal planned leadtimes at each stage. Different from Yano (1987a) and Gong et al. (1996), Elhafsi (2002) penalizes tardiness of intermediate stages, while in the former ones the penalty cost is only incurred if the last stage is late. Elhafsi (2002) proposes a recursive algorithm to compute the expected cost and solves the resulting convex nonlinear optimization problem to determine the optimal planned leadtimes.

Research on setting the planned leadtimes for assembly systems has been initiated by Yano $(1987 b)$. The author considers a system with two subassemblies and a final assembly. The system incurs inventory holding costs for each stage from the moment the stage starts until the product is delivered to the customer. If the product is available after the promised delivery date, a penalty cost is charged per unit late. The objective is to find the optimal planned leadtimes. Yano (1987b) formulates the problem as a non-linear program. Although the cost function is not convex in all planned leadtimes, it has some properties that enable Yano 1987b) to solve the problem numerically. For systems with more than three stages, this approach often leads to computational problems.

After the seminal work by Yano (1987b) researchers developed approximation methods to solve the leadtime optimization problem for larger assembly systems. Hopp \& Spearman (1993) present a model to determine the optimal leadtimes for purchasing components for a manufacturing system which performs the final assembly of these components. The authors develop an iterative approximation procedure to determine the optimal purchasing leadtimes. Shore (1995) studies the same problem and proposes an alternative procedure that results in closed-form expressions for the decision variables. Song et al. (2001) develop a recursive procedure to estimate the distributions of stage leadtimes and propose a method to calculate stage due dates so that a specific service target is met. Considering cost minimization as in Yano (1987b), Axsäter (2005) studies a multi-echelon assembly system and suggests a decomposition technique to set the stage start times. Different from Axsäter (2005), Chauhan et al. (2009) study a single-period setting. The authors develop an approximation procedure to set the stage start times. In Ben-Ammar et al. (2018) planned leadtimes for multi-level assembly systems are determined. The authors relax specific assumptions on cost parameters and develop a Branch and Bound algorithm to approximate optimal planned leadtimes. 
In another recent study, Atan et al. (2016) study an assembly system that consists of a number of parallel multi-process stages feeding a multi-process final assembly stage. Each process has a stochastic leadtime. From the moment a production process starts until the final product is delivered to the customer, the system incurs marginal process holding cost and a penalty cost is charged for late deliveries to the customer. The authors derive recursive equations for the tardiness and earliness of all processes and determine an exact expression for the total expected cost. They develop an iterative heuristic procedure to calculate the optimal planned leadtimes. This procedure is based on a conjecture which claims that the probability that a process is responsible for the lateness of the overall system is proportional to the value added by that process.

Project scheduling requires setting planned finish times for activities in a project. This is related to the problem studied in this paper. The literature on project scheduling is extensive. Multiple methods with different objectives and solutions have been proposed. Among the most relevant studies to our work is Trietsch (2006). The author studies a project scheduling problem with random activity durations. The objective is to set time buffers between activities, so that the sum of the activity holding costs and penalty costs due to the lateness of the project is minimized. Trietsch (2006) shows that the optimal buffer time for an activity is proportional to the holding cost of the activity. Trietsch \& Baker (2012) implement the optimization method of Trietsch (2006) in a decision support system for project planning.

Based on similarity in modeling approaches, we believe that project management problems can benefit from the results of studies on leadtime planning and vice versa. Hence, an important contribution of our paper is the identification of different application areas that use a different language in formulating and solving similar problems. Therefore, we do not only contribute to the literature on leadtime planning but also to the literature on project management.

\section{Problem Formulation}

We consider a configure-to-order assembly system consisting of $N$ parallel stages delivering subassemblies to a single final assembly stage. The set of subassembly stages is $V=\{1,2, \ldots, N\}$ and 0 is the final assembly stage (see Figure 1). Subassembly processes are initiated by a customer demand for a unit of product and the product is ready for delivery at completion of stage 0 . Each stage $i \in\{0\} \cup V$ requires execution of multiple tasks. We refer to the total time required to finish all tasks within a stage as the leadtime of this stage. Non-negative random variable $T_{i}$ with cumulative distribution $F_{i}(\cdot)$ and density $f_{i}(\cdot)$ represents the leadtime of stage $\left\{1\right.$. We assume that $f_{i}\left(t_{i}\right)>0$ for all $t_{i} \geq 0$ and leadtimes at different stages are independent. The results in the rest of the paper remain valid

\footnotetext{
${ }^{1}$ Unless otherwise stated, all definitions are valid for $i \in\{0\} \cup V$
} 


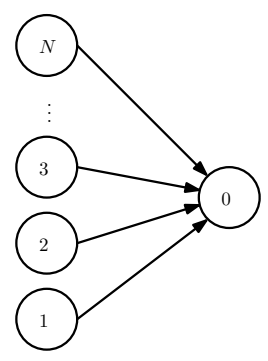

Figure 1: An assembly system with $N$ subassemblies and one final stage

for some specific cases of dependent leadtimes. Identifying these specific cases is among our future research plans.

The system operates according to a plan that assigns a leadtime to each stage. This leadtime is called the planned leadtime. The planned leadtime of stage $i$ is $x_{i}$. We define $s_{i}$ as the planned start time of stage $i$. Without loss of generality, we set the planned customer delivery time to 0 . Then the planned start times can be easily calculated from the planned leadtimes as

$$
\begin{aligned}
& s_{0}=-x_{0}, \\
& s_{i}=-x_{i}-x_{0} \quad i \in V .
\end{aligned}
$$

The actual start time of a stage is a random variable and depends on the actual leadtimes of all predecessors. For all stages with no predecessor stage, i.e. for all $i \in V$, we assume that all required resources are available at the planned start time and thus these stages can always start on time. However, their completion times are random. If $T_{i}=x_{i}$, then stage $i$ is on time. Otherwise, it is either early or late. We define $E_{i}$ and $L_{i}$ as the earliness and lateness of stage $i$, respectively. Let $(x)^{+}=\max \{0, x\}$. Then, for all $i \in V$,

$$
\begin{aligned}
& E_{i}=\left(x_{i}-T_{i}\right)^{+} \\
& L_{i}=\left(T_{i}-x_{i}\right)^{+} .
\end{aligned}
$$

Stage 0 can only start after all subassemblies have been delivered. If one or more subassemblies are late, production at stage 0 starts immediately after the latest subassembly has been finished. If all subassemblies finish early, stage 0 starts at its planned start time $s_{0}$. This assumption of holding back production when predecessor stages finish early is common in the literature (Yano 1987a, Axsäter 2005). It represents the situation where materials and workforce are available at the planned start time, and not earlier. Hence, the earliness and lateness expressions for stage 0 are

$$
\begin{aligned}
& E_{0}=\left(x_{0}-T_{0}-\max _{i \in V}\left\{L_{i}\right\}\right)^{+}, \\
& L_{0}=\left(T_{0}+\max _{i \in V}\left\{L_{i}\right\}-x_{0}\right)^{+} .
\end{aligned}
$$


We define $W_{i}$ as the waiting time of stage $i$ due to the lateness of other parallel stages,

$$
W_{i}=\max _{j \in V}\left\{L_{j}\right\}-L_{i}, \quad i \in V .
$$

Since $W_{0}$ has no parallel stages, $W_{0}=0$. Note that the random variables $E_{i}, L_{i}$ and $W_{i}$ depend on the vector of decision variables $\mathbf{x}=\left\{x_{0}, x_{1}, \ldots, x_{N}\right\}$. We do not explicitly indicate this dependence in the notation, except when it improves readability (as in Lemma 1, where we write $L_{0}(\mathbf{x})$ ). For a

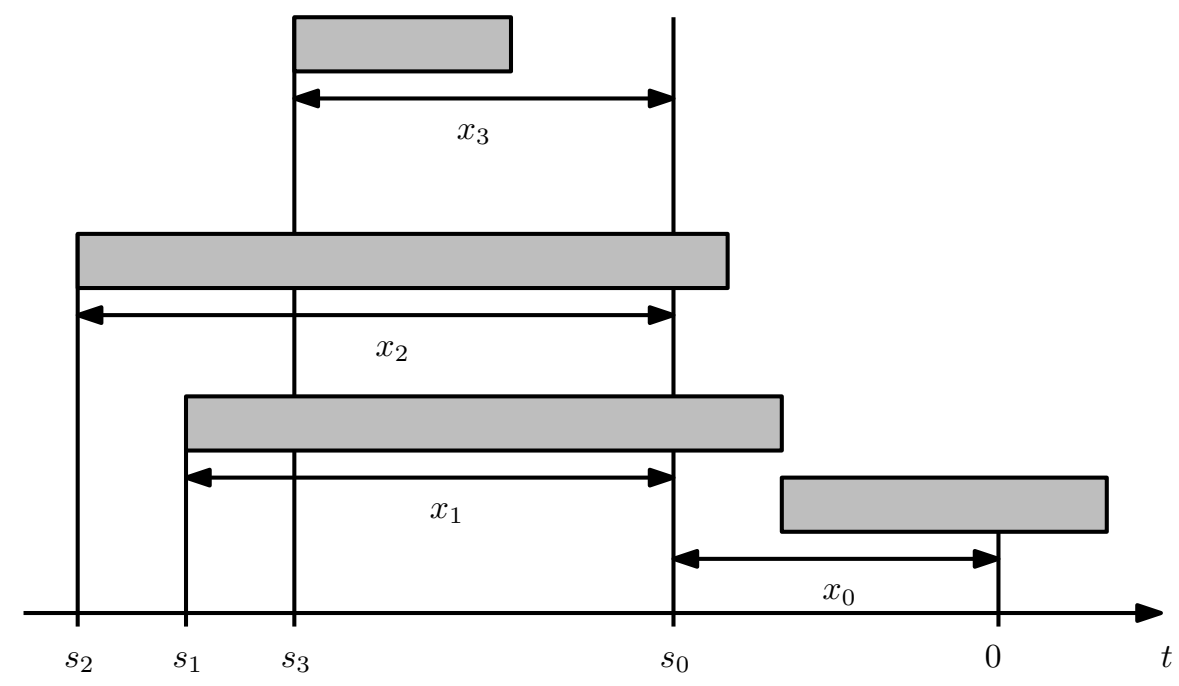

Figure 2: Timeline for a realization of a production plan in a four-stage assembly system

four stage assembly system, a production plan and a realization of leadtimes are shown in Figure 2.

A realization of the random variables $T_{0}, T_{1}, \ldots, T_{N}$ is indicated by $\omega=\left(t_{0}, t_{1}, \ldots, t_{N}\right) \in \Omega=\mathbb{R}_{+}^{N+1}$. In particular, $T_{i}(\omega)=t_{i}$ is a realization of $T_{i}$.

The timeline in Figure 2 shows the planned start and finish time for each stage. The gray horizontal bars denote the realizations $T_{i}(\omega)$ of the random variables $T_{i}$. In this case, stage 1 and 2 finish late, while stage 3 finishes early. Stage 1 is the latest one and subassemblies produced at stage 2 and 3 have to wait for stage 1 . The final assembly stage starts at $s_{0}+L_{1}(\omega)$ and finishes later than its planned finish time. Thus the final product is delivered late.

The system incurs a marginal holding $\operatorname{cost} h_{i}>0$ from the planned start time of stage $i$ until the final product is delivered to the customer. The total holding costs per unit time at stage $i$ are $h_{i}^{c}$. We have $h_{i}^{c}=h_{i}$ for $i \in V$ and $h_{0}^{c}=\sum_{i \in V} h_{i}^{c}+h_{0}$. In addition to the holding costs, the system incurs a penalty cost $p$ per unit time late for delivery to the customer. The total holding cost for the planned duration of stage $i$ is $h_{i}^{c} x_{i}$ for $i \in\{0\} \cup V$. If the product is delivered late, in addition to the penalty cost, a holding cost $h_{0}^{c}$ is also charged per unit late. Hence, the total costs for lateness of the final stage are $\left(h_{0}^{c}+p\right) L_{0}$. Let $\mathbf{x}=\left(x_{0}, \ldots, x_{N}\right)$ be the vector of all planned leadtimes. We 
define $C(\mathbf{x})$ as the total expected cost of a product produced according to the planned leadtimes $\mathbf{x}$. Then

$$
C(\mathbf{x})=\sum_{i=0}^{N} h_{i}^{c} x_{i}+\left(h_{0}^{c}+p\right) \mathbb{E}\left[L_{0}\right] .
$$

The cost $C(\mathbf{x})$ is composed of two terms. The first term is the deterministic term of planned leadtimes. The second one is the variability term depending on stochastic and planned leadtimes. The objective is to determine the planned leadtimes that minimize the total expected $\operatorname{cost} C(\mathbf{x})$.

\section{Solution Approach}

Our solution approach is based on solving a set of Newsvendor equations. We show that the probability that a stage is "blamed" for the lateness of the system equals a Newsvendor fractile. We first explain the concept of blaming by defining a blame policy in Section 4.1 and then derive the corresponding Newsvendor equations for our cost function in Section 4.2 .

\subsection{Blame Policy}

When a product is delivered late, one may ask which stage or stages caused the lateness. For each late delivery, there are one or more stages with leadtimes exceeding their planned leadtimes. These stages are candidates to be blamed. In this section, we define a blame policy to identify the single stage to be blamed for the lateness of the system for a given realization of the random leadtimes. In other words, for each possible event that results in lateness of the system, the blame policy identifies which stage is blamed for the lateness.

Next, we define a set of rules, referred as blame conditions. We define for each stage $i \in\{0\} \cup V$ event $A_{i}$ as the set of realizations $\omega=\left(t_{0}, t_{1}, \ldots, t_{N}\right)$ satisfying the following blame conditions:

1. Stage $i$ starts at its planned start time.

2. The leadtime $T_{i}(\omega)$ of stage $i$ exceeds its planned leadtime, so $T_{i}(\omega)>x_{i}$.

3. The successor of stage $i$ is able to start immediately after stage $i$ finishes, so $W_{i}(\omega)=0$.

4. The final product is delivered late; $L_{0}(\omega)>0$.

More formally,

$$
\begin{aligned}
& A_{0}=\left\{\omega: \max _{j \in V}\left\{L_{j}(\omega)\right\}=0, L_{0}(\omega)>0\right\} \\
& A_{i}=\left\{\omega: T_{i}(\omega)>x_{i}, \max _{j \in V}\left\{L_{j}(\omega)\right\}=L_{i}(\omega), L_{0}(\omega)>0\right\}, \quad i \in V .
\end{aligned}
$$


Event $A_{i}$ represents the set of all realizations $\omega$ in which stage $i$ is blamed for late delivery. Note that a stage is only blamed when the final product is late $\left(L_{0}(\omega)>0\right)$. If a subassembly stage finishes late, but this lateness is compensated by the final stage, none of the stages is blamed. Also, if both subassembly stage $i$ and stage 0 exceed their planned leadtimes, $T_{i}(\omega)>x_{i}$ and $T_{0}(\omega)>x_{0}$, only stage $i$ is blamed. Stage 0 can only be blamed if it starts on time, that is $L_{i}(\omega)=0$ for all $i \in V$. Finally, since we assume the random variable $T_{i}$ to be continuous, the events $A_{i}$ are mutually exclusive for all $i \in\{0\} \cup V$ and together cover all possible realizations $\omega=\left(t_{0}, t_{1}, \ldots, t_{N}\right)$ for which delivery is late. For all $i \in\{0\} \cup V$ we define $\Omega$ as the supporting probability space of $P\left(A_{i}\right)$ and hence it follows that $P\left(L_{0}>0\right)=\sum_{i \in\{0\} \cup V} P\left(A_{i}\right)$.

For a given realization of the random variables, the blame policy identifies the specific stage that is responsible for the late completion of the final product. As an example, consider the realization in Figure 2, The stage to be blamed is stage 1, because it starts on time, exceeds its planned leadtime, stage 0 starts immediately after stage 1 finishes and delivery is late. Stage 2 also exceeds its planned leadtime, but cannot be blamed, since stage 0 starts after stage 1 finishes, i.e., Condition 3 is violated. Stage 3 cannot be blamed because conditions 2 and 3 are violated, $T_{3}(\omega)<x_{3}$ and $W_{3}(\omega)>0$. Stage 0 cannot be blamed, because it starts later than planned and thus Condition 1 is violated.

It is crucial to note that the blame policy is independent of the cost function. It can be applied to all planned leadtime optimization problems for any assembly system structure with stochastic leadtimes. As it will be seen in the rest of paper, we do not use this policy to obtain the optimal planned leadtimes. It is possible to define other blame policies. For example, one blame policy might be that no matter what happens with the leadtimes of the subassemblies, the final assembly is always responsible for the lateness of the system. The optimal planned leadtimes under this alternative blame policy and our blame policy are the same. Note that our policy is fairer compared to this alternative policy. What makes our blame policy special and different from its alternatives is that, the optimality equations, i.e., equations satisfied by the optimal planned leadtimes, are Newsvendor equations. In addition, their Newsvendor equations result in a unique solution. We provide the details in Section 4.2 .

\subsection{Newsvendor Equations}

Newsvendor equations originate from the field of inventory management. A Newsvendor equation describes the optimal stockout probability and it can be used to calculate the optimal order-up-to level. At optimality, penalty and inventory holding costs are balanced. For our problem, Newsvendor equations describe the probability that a specific stage is blamed for late delivery. The optimal planned leadtimes can be obtained by solving these Newsvendor equations. The optimal solution balances holding costs for being early and penalty cost for being late. Summing these Newsvendor 
equations over all stages results in an equation describing the probability that the product is delivered late. We call this "the general Newsvendor equation."

We define $\mathbf{x}^{*}$ as the optimal planned leadtime solution, where superscript $*$ is used to denote state variables at optimality. For example, $L_{i}^{*}=\left(T_{i}-x_{i}^{*}\right)^{+}$is the lateness at stage $i$ when the planned leadtimes are set to their optimal values. Lemma 1 presents the partial derivatives of $\mathbb{E}\left[L_{0}\right]$. The proof of this lemma and those of all other results in this paper are deferred to the Appendix.

\section{Lemma 1.}

$$
\frac{\partial \mathbb{E}\left[L_{0}(\mathbf{x})\right]}{\partial x_{0}}=-\sum_{i=0}^{N} P\left(A_{i}\right), \quad \frac{\partial \mathbb{E}\left[L_{0}(\mathbf{x})\right]}{\partial x_{i}}=-P\left(A_{i}\right), \quad i \in V .
$$

From (1) and Lemma 1 it follows that the gradient of $C(\mathbf{x})$ is given by

$$
\begin{aligned}
\nabla C(\mathbf{x}) & =\left(\frac{\partial C(\mathbf{x})}{\partial x_{0}}, \ldots, \frac{\partial C(\mathbf{x})}{\partial x_{N}}\right) \\
& =\left(h_{0}^{c}-\left(h_{0}^{c}+p\right) \sum_{i=0}^{N} P\left(A_{i}\right), h_{1}^{c}-\left(h_{0}^{c}+p\right) P\left(A_{1}\right), \ldots, h_{N}^{c}-\left(h_{0}^{c}+p\right) P\left(A_{N}\right)\right) .
\end{aligned}
$$

$\nabla C(\mathbf{x})$ vanishes at optimality. Hence, we obtain the following optimality equations, i.e., equations that are satisfied by the optimal solution $\mathbf{x}^{*}$

$$
P\left(A_{i}\right)=\frac{h_{i}^{c}}{h_{0}^{c}+p}=\frac{h_{i}}{h_{0}^{c}+p}, \quad i \in V
$$

and

$$
\sum_{i=0}^{N} P\left(A_{i}\right)=\sum_{i=0}^{N} \frac{h_{i}}{h_{0}^{c}+p}=\frac{h_{0}^{c}}{h_{0}^{c}+p} .
$$

The left-hand side of (4) is the probability that the product is delivered late, that is, $P\left(L_{0}^{*}>0\right)$. This equation is referred to as the general Newsvendor equation. Subtracting (3) from (4) yields

$$
P\left(A_{0}\right)=\frac{h_{0}}{h_{0}^{c}+p} .
$$

These findings are summarized in the next theorem stating that at optimality, the probability that stage $i$ is blamed for late delivery is proportional to the value $h_{i}$ this stage adds to the final product.

Theorem 1. The optimal planned leadtime solution $\boldsymbol{x}^{*}$ of the unconstrained optimization problem $\min _{\boldsymbol{x}} C(\boldsymbol{x})$ satisfies the following set of Newsvendor equations:

$$
P\left(A_{i}\right)=\frac{h_{i}}{h_{0}^{c}+p}, \quad i \in\{0\} \cup V .
$$

Clearly, if $h_{i}$ is high, it is costly to finish stage $i$ early as holding cost will accumulate. Hence, under the optimal solution, stages which add high value to the final product are planned such that, with high probability, they are to be blamed for the late delivery. 
The blame probabilities in Theorem 1 can be formulated in terms of the leadtime densities and decision variables $\mathbf{x}$. For stage $i \in V$ we have

$$
P\left(A_{i}\right)=P\left(T_{i}>x_{i}, W_{i}=0, L_{0}>0\right),
$$

where the random variables $W_{i}$ and $L_{0}$ depend on the leadtime $T_{i}$. Conditioning on $T_{i}$ yields

$$
\begin{aligned}
P\left(A_{i}\right) & =\int_{x_{i}}^{\infty} P\left(W_{i}=0, L_{0}>0 \mid T_{i}=t_{i}\right) f_{i}\left(t_{i}\right) d t_{i} \\
& =\int_{x_{i}}^{\infty} P\left(\max _{j \in V}\left\{L_{j}\right\}=L_{i}, L_{0}>0 \mid T_{i}=t_{i}\right) f_{i}\left(t_{i}\right) d t_{i} \\
& =\int_{x_{i}}^{\infty} \prod_{j=1, j \neq i}^{N} P\left(T_{j}-x_{j}<t_{i}-x_{i}\right) P\left(t_{i}-x_{i}+T_{0}-x_{0}>0\right) f_{i}\left(t_{i}\right) d t_{i} \\
& =\int_{x_{i}}^{\infty} \prod_{j=1, j \neq i}^{N}\left(\int_{0}^{x_{j}+t_{i}-x_{i}} f_{j}\left(t_{j}\right) d t_{j}\right)\left(\int_{x_{0}-\left(t_{i}-x_{i}\right)}^{\infty} f_{0}\left(t_{0}\right) d t_{0}\right) f_{i}\left(t_{i}\right) d t_{i} .
\end{aligned}
$$

For stage 0 we get

$$
\begin{aligned}
P\left(A_{0}\right) & =P\left(\max _{j \in V}\left\{L_{j}\right\}=0, L_{0}>0\right) \\
& =\prod_{j=1}^{N} P\left(T_{j}-x_{j}<0\right) P\left(T_{0}-x_{0}>0\right) \\
& =\prod_{j=1}^{N}\left(\int_{0}^{x_{j}} f_{j}\left(t_{j}\right) d t_{j}\right) \int_{x_{0}}^{\infty} f_{0}\left(t_{0}\right) d t_{0} .
\end{aligned}
$$

The above expressions can be used to numerically solve the optimal planned leadtimes from the Newsvendor equations in Theorem 1 .

\section{$5 \quad$ Structural Results}

Our cost function fundamentally differs from cost functions studied in the literature. This has implications for the structure of the optimal solution. In Section 5.1, we describe properties of our cost function and optimal solution. Then we compare our optimal solution with the one obtained by Atan et al. (2016) in Section 5.2.

\subsection{Properties of the Cost Function and the Optimal Solution}

Since $\min _{\mathbf{x}} C(\mathbf{x})$ is an unconstrained optimization problem, one or more planned leadtimes of the optimal solution may be negative. For cost functions studied in the literature, this turns out to be 
possible, as shown in Section 6.2. Negative planned leadtimes, however, are often not accepted in practice: it is counter-intuitive to have the planned finish time before the planned start time of a task. The next theorem states that, for our cost function, vectors $\mathbf{x}$ with negative planned leadtimes are never optimal.

Theorem 2. For any $\mathbf{x}$ with at least one negative planned leadtime $x_{i}, i \in\{0\} \cup V$, there exists a vector of non-negative planned leadtimes with lower cost.

This theorem implies that the optimization problem can be restricted to $\mathbf{x} \geq 0$. Since $C(\mathbf{x}) \geq$ $\sum_{i=0}^{N} h_{i}^{c} x_{i}$, the cost of the all zero vector $\mathbf{0}$ is less than the cost of any $\mathbf{x}$ with $\sum_{i=0}^{N} h_{i}^{c} x_{i}>C(\mathbf{0})$. Hence, optimization can be further restricted to the bounded region $\left\{\mathbf{x}: \mathbf{x} \geq 0, \sum_{i=0}^{N} h_{i}^{c} x_{i} \leq C(\mathbf{0})\right\}$. Since $C(\mathbf{x})$ is continuous, we can conclude that the optimal $\mathbf{x}^{*}$ indeed exists.

Proposition 1. The optimal solution $\mathbf{x}^{*}$ of $\min _{\boldsymbol{x}} C(\boldsymbol{x})$ exists and is non-negative.

Using that the solution space can be restricted to non-negative planned leadtimes, we next derive monotonicity properties for the blame probabilities of different stages. Lemma 2 holds for any $i \in V$, while Lemma 3 holds for stage 0 .

Lemma 2. For stage $i \in V$ and $\mathbf{x} \geq 0$, the probability $P\left(A_{i}\right)$ is strictly decreasing in $x_{i}$ and $x_{0}$ and strictly increasing in $x_{j}, j \in V, j \neq i$.

Lemma 3. For stage 0 and $\mathbf{x} \geq 0$, the probability $P\left(A_{0}\right)$ is strictly decreasing in $x_{0}$ and strictly increasing in $x_{i}, i \in V$.

According to the above lemmas, blame probability $P\left(A_{i}\right)$ is decreasing in $x_{0}$ and $x_{i}$ and increasing in any other planned leadtime. Hence, final stage 0 is the only stage for which a larger planned leadtime $x_{0}$ leads to a reduction of all blame probabilities.

Theorem 1 states that the optimal solution $\mathrm{x}^{*}$ satisfies a set of Newsvendor equations. The monotonicity results in Lemma 2,3 are instrumental to establish the following theorem.

Theorem 3. The Newsvendor equations (6) have a unique non-negative solution.

Corollary 1. The optimal solution $\mathbf{x}^{*}$ of $\min _{\boldsymbol{x}} C(\boldsymbol{x})$ is unique.

\subsection{Comparison with an Alternative Cost Function}

As explained in Section 1, our cost function charges the holding costs from the planned start time of the stages while in other studies the holding cost is charged only after the stages actually start. 
Except for this difference in the cost function, the modeling assumptions in Atan et al. (2016) are exactly the same as ours. In this section, we provide an analytical comparison of the cost functions.

Let $C^{a}(\mathbf{x})$ denote the total expected cost used in Atan et al. (2016). The expression for $C^{a}(\mathbf{x})$ is

$$
C^{a}(\mathbf{x})=\sum_{i=0}^{N} h_{i}^{c} x_{i}-h_{0} \mathbb{E}\left[\max _{i \in V}\left\{L_{i}\right\}\right]+\left(h_{0}^{c}+p\right) \mathbb{E}\left[L_{0}\right]
$$

The only difference between (1) and (8) is the second term of the cost function $C^{a}(\mathbf{x})$. This term contains the random variable $\max _{i \in V}\left\{L_{i}\right\}$, which is the lateness of the latest subassembly. Atan et al. (2016) exclude the expected holding cost that might be incurred at stage 0 during the time it waits for all subassemblies to finish

At first sight, the new cost function looks simpler. However, it is more challenging to solve. Atan et al. (2016) rely on the fact that, under their accounting scheme the optimality equations are decoupled. This is why, they can use a recursive procedure to solve for the optimal planned leadtimes. On the other hand, under the new accounting scheme, the optimality equations are not decoupled. Therefore, we cannot use the recursive procedure to solve for the optimal planned leadtimes. This is why a new solution procedure is required.

We define $\mathbf{x}^{\mathbf{a} *}$ as the optimal solution to the optimization problem $\min _{\mathbf{x}} C^{a}(\mathbf{x})$. For any planned leadtime vector $\mathbf{x}$ we can compute the expected costs using (1) and (8). Since our cost function penalizes the lateness of intermediate time points while $C^{a}(\mathbf{x})$ does not, our function always leads to higher expected costs. This result is stated in the following proposition:

Proposition 2. For the cost functions $C(\cdot)$ and $C^{a}(\cdot)$ the following inequalities hold:

(a) $C(\mathbf{x}) \geq C^{a}(\mathbf{x})$ for all $\mathbf{x}$;

(b) $C\left(\mathbf{x}^{*}\right) \geq C^{a}\left(\mathbf{x}^{\mathbf{a} *}\right)$.

Obviously, $\mathbf{x}^{*}$ and $\mathbf{x}^{\mathbf{a} *}$ are not necessarily the same. The next proposition compares the two optimal planned leadtimes.

Proposition 3. For the optimal planned leadtime solutions $\mathbf{x}^{*}$ and $\mathbf{x}^{\mathbf{a} *}$ the following relations hold:

(a) $x_{0}^{*}<x_{0}^{a *}$ and $x_{i}^{*}>x_{i}^{a *}$ for all $i \in V$;

(b) There exist $i \in V$ such that $x_{i}^{*}+x_{0}^{*} \geq x_{i}^{a *}+x_{0}^{a *}$.

Proposition 3(a) states that our solution $\mathbf{x}^{*}$ allocates more time to each of the subassembly stages and allocates less time to the final assembly stage compared to $\mathbf{x}^{a *}$. As a consequence, the solution $\mathbf{x}^{*}$ ensures that the intermediate deadline is met more often. This is in line with the intended practical 
applications of both solutions. Solution $\mathrm{x}^{*}$ should be used when exceeding the intermediate deadline is costly because materials are waiting. Solution $\mathrm{x}^{\mathbf{a} *}$ should be used when materials are delivered JIT. Part (b) implies that for a two-stage serial system (i.e., $N=1$ ), the total leadtime is longer for our solution. This is an intuitive result, since safety time added to a subassembly stage cannot compensate for lateness in the final assembly, while safety time in the final assembly can compensate for lateness in both the subassembly and the final assembly.

The difference between our expected cost and the one in Atan et al. (2016) is $h_{0} \mathbb{E}\left[\max _{i \in V}\left\{L_{i}\right\}\right]$. Hence, an important parameter driving differences between $\mathrm{x}^{*}$ and $\mathrm{x}^{\mathbf{a} *}$ is $h_{0}$. Below we study for a two-stage serial system the asymptotic behavior of the optimal planned leadtimes as $h_{0} \rightarrow \infty$, while keeping service level $\alpha$ constant. The service level is defined as

$$
\alpha=P\left(L_{0}=0\right)=\frac{p}{h_{0}^{c}+p}
$$

As $h_{0}$ changes, we keep service level $\alpha$ constant by adapting $p=\frac{\alpha}{1-\alpha} h_{0}^{c}$ (and not changing $h_{1}$ ).

Proposition 4. For a two-stage serial system, as $h_{0} \rightarrow \infty$ while keeping service level $\alpha$ constant:

$$
\begin{aligned}
\lim _{h_{0} \rightarrow \infty}\left\{x_{0}^{a *}\right\} & =F_{0}^{-1}(\alpha), \\
\lim _{h_{0} \rightarrow \infty}\left\{x_{1}^{a *}\right\} & =\infty, \\
\lim _{h_{0} \rightarrow \infty}\left\{x_{0}^{*}\right\} & =F_{0}^{-1}(\alpha), \\
\lim _{h_{0} \rightarrow \infty}\left\{x_{1}^{*}\right\} & =\infty .
\end{aligned}
$$

We see that the asymptotic behavior of both optimal solutions is similar. The optimal planned leadtimes of the final stage approach the same limiting value. The leadtimes of the first stage grow without bound, which is due to the blame probability $P\left(A_{1}\right)=\frac{h_{1}}{h_{0}^{c}+p}$ approaching to 0 . Hence, in the limit, lateness of the complete system is equal to lateness of the final stage. The above proposition does not provide information on the asymptotic behavior of the difference between the optimal planned leadtimes of the first stage. Numerical experiments indicate that $x_{1}^{*}-x_{1}^{a *}$ does converge, though not to 0 (as for the final stage) but to a positive number.

We obtain additional analytical results for the case with exponentially distributed leadtimes. We consider a two-stage system. In the following propositions we provide the optimality equations for both cost functions.

Proposition 5. For a two-stage serial system with independent and identically distributed exponential leadtimes with rate $\lambda$, the optimal solution $\mathbf{x}^{*}$ satisfies the following set of Newsvendor equations:

$$
\begin{aligned}
\left(1+\lambda x_{0}^{*}\right) e^{-\lambda\left(x_{0}^{*}+x_{1}^{*}\right)} & =\frac{h_{1}}{h_{0}^{c}+p} \\
e^{-\lambda x_{0}^{*}}-e^{-\lambda\left(x_{0}^{*}+x_{1}^{*}\right)} & =\frac{h_{0}}{h_{0}^{c}+p}
\end{aligned}
$$


Proposition 6. For a two-stage serial system with independent and identically distributed exponential leadtimes with rate $\lambda$, the optimal solution $\mathrm{x}^{\mathbf{a} *}$ satisfies the following set of Newsvendor equations:

$$
\begin{aligned}
\lambda x_{0}^{a *} e^{-\lambda\left(x_{1}^{a *}+x_{0}^{a *}\right)} & =\frac{h_{1}}{h_{0}^{c}+p} \\
e^{-\lambda x_{0}^{a *}} & =\frac{h_{0}}{h_{0}^{c}+p}
\end{aligned}
$$

The expressions in Proposition 6 suggests that we can obtain $x_{0}^{a *}$ and $x_{1}^{a *}$ recursively. On the other hand, the expressions in Proposition 5 are not decoupled. Hence, under our cost accounting scheme, obtaining the optimal solution is more challenging.

In addition to the optimality equations, for the special case with exponential leadtimes, we can determine the expression for the difference $x_{1}^{*}-x_{1}^{a *}$ as $h_{0} \rightarrow \infty$.

Proposition 7. For a two-stage serial system with independent and identically distributed exponential leadtimes with rate $\lambda$, we have

$$
\lim _{h_{0} \rightarrow \infty}\left\{x_{1}^{*}-x_{1}^{a *}\right\}=\frac{1}{\lambda}\left(\ln \left(\lambda x_{0}^{*}+1\right)-\ln \left(\lambda x_{0}^{*}\right)\right) .
$$

\section{$6 \quad$ Numerical Analysis}

Section 5.2 outlines differences between our optimal solution and the one in Atan et al. (2016). In this section we provide the numerical results which do not only quantify differences in optimal costs but also structural differences in the optimal solutions.

\subsection{Cost Comparison}

In this section we provide a representative example to show differences between the optimal solutions and the corresponding optimal costs. We consider an assembly system with two subassemblies (stage 1 and 2) and a final assembly stage (stage 0). We assume equal echelon unit holding costs for all stages, thus $h_{0}=h_{1}=h_{2}=1$. We set the unit penalty costs to $p=27$. Hence, under the optimal solution, the service level is $\alpha=\frac{p}{h_{0}^{c}+p}=90 \%$. We assume that leadtimes are exponentially distributed with rate $\lambda_{i}=1$ for all $i \in\{0\} \cup V$. Table 1 provides the optimal planned leadtimes and some properties of the optimal solutions and Table 2 summarizes the expected total costs.

The expected costs for each of the optimal leadtime solutions $\mathbf{x}^{*}$ and $\mathbf{x}^{\mathbf{a} *}$ can be computed according to both cost functions $C(\cdot)$ and $C^{a}(\cdot)$. These are also shown in Table 2. Suppose a company uses cost function $C^{a}(\cdot)$ and determines the optimal solution $\mathbf{x}^{\mathbf{a} *}$. The total expected cost 


\begin{tabular}{|c|c|c|c|c|c|}
\hline & $x_{0}$ & $x_{1}, x_{2}$ & Total & $P\left(\max \left\{L_{1}, L_{2}\right\}>0\right)$ & $P\left(L_{0}>0\right)$ \\
\hline $\mathbf{x}^{*}$ & 3.01 & 1.73 & 4.75 & 0.32 & 0.1 \\
\hline $\mathbf{x}^{\mathbf{a} *}$ & 3.40 & 1.18 & 4.58 & 0.52 & 0.1 \\
\hline
\end{tabular}

Table 1: Structural differences between the two optimal planned leadtime solutions.

\begin{tabular}{|c|c|c|c|}
\hline$C\left(\mathbf{x}^{*}\right)$ & $C^{a}\left(\mathbf{x}^{*}\right)$ & $C\left(\mathbf{x}^{\mathbf{a} *}\right)$ & $C^{a}\left(\mathbf{x}^{\mathbf{a} *}\right)$ \\
\hline 16.03 & 15.69 & 16.18 & 15.61 \\
\hline
\end{tabular}

Table 2: Expected costs under the optimal planned leadtime solutions.

for this solution is 15.61. However, this cost function does not take into account costs that occur if stage 0 cannot start in time. Cost function $C(\cdot)$ does take these additional costs into account. Given that the production is planned according to solution $\mathbf{x}^{\mathbf{a} *}$, the expected cost according to cost function $C(\cdot)$ will be 16.18 . This is an increase of $3.6 \%$. Thus, for this case, cost function $C^{a}(\cdot)$ neglects a significant part of the total expected costs. However, the solution $\mathrm{x}^{\mathbf{a} *}$ is not optimal for cost function $C(\cdot)$. If a company would optimize according to this cost function, the solution is $\mathbf{x}^{*}$. This leads to a total costs of 16.03 , which implies an improvement of $0.9 \%$.

One could argue that an improvement of $0.9 \%$ in expected costs is relatively small and thus that both solutions $\mathbf{x}^{\mathbf{a} *}$ and $\mathbf{x}^{*}$ can be used. There are a few reasons why the cost difference is this small. First, the holding cost during the average duration of each stage, i.e., $\sum_{i=0}^{N} h_{i}^{c} \mathbb{E}\left[T_{i}\right]$, appears in both cost functions and it is constant. In this example, the value of this constant term is 5.0, i.e., almost one third of the total costs. Second, the cost for being late at the final stage are $\left(h_{0}^{c}+p\right) \mathbb{E}\left[L_{0}\right]$. A high service level requirement, such as $90 \%$, makes this term significant. Under solution $\mathbf{x}^{*}$ this term equals 3.52 (22.0\% of the total costs), while under solution $\mathrm{x}^{\mathbf{a} *}$ this term equals 3.61 (22.3\% of the total costs).

Although the costs for both solutions are close, there are significant differences in the structure of the optimal solutions. In Table 1, we provide the planned leadtimes of each stage and lateness probabilities. We observe that $\mathrm{x}^{*}$ allocates significantly less time to the final stage (3.01 vs 3.40). Since stages 1 and 2 have equal leadtime distributions and equal holding costs, these stages have the same optimal planned leadtimes in both solutions. We see that $\mathbf{x}^{*}$ plans more time for the subassembly stages (1.73 versus 1.18 ), a difference of $46 \%$. This has a significant impact on the probability that one or more subassemblies finish late. This probability is 0.32 if $\mathbf{x}^{*}$ is used while it is 0.52 if $\mathbf{x}^{\mathbf{a} *}$ is used. Hence, the probability that the final assembly can start in time is 0.68 for our solution. This probability is 0.48 for the other solution. Therefore, the advantage of using $\mathbf{x}^{*}$ instead of $\mathbf{x}^{\mathbf{a} *}$ is that intermediate deadlines are met more often, while expected costs and total 
leadtime only change marginally. From a production planner's perspective, this is useful, since it makes scheduling resources for different products easier.

The insights of this numerical example can be generalized to other parameter combinations. The main driver in the cost difference between our solution and the one by Atan et al. $(2016)$ is the relative importance of meeting the intermediate deadline (start of final assembly) compared to the final deadline (delivery to the customer). A high $h_{0} /\left(h_{1}+h_{2}\right)$ ratio makes the intermediate deadline important, while a high $p / h_{0}^{c}$ ratio makes the final deadline important. Increasing $h_{0}$ while keeping other parameters constant increases the importance of the intermediate deadline and decreases the importance of the final deadline. This leads to higher cost differences. However, in such an example, the service level would decrease significantly. For example, when $h_{1}=h_{2}=1, h_{0}=10$ and $p=27$, the optimal service level is $27 / 39=69 \%$.

Similarly, increasing $p$ while keeping other parameters constant makes the final deadline important and reduces the cost difference between our solution and the one by Atan et al. (2016). To show this, we perform a numerical experiment in which we vary $p$. We consider a two-stage serial system with exponentially distributed leadtimes: $\lambda_{1}=\lambda_{0}=1$. Holding cost parameters are $h_{1}=h_{0}=1$. We calculate the optimal solutions $\mathbf{x}^{*}$ and $\mathbf{x}^{\mathbf{a} *}$ and the corresponding expected $\operatorname{cost} C(\mathbf{x} *)$ and $C^{a}\left(\mathbf{x}^{\mathbf{a} *}\right)$. In Figure 3 the relative cost difference, defined as $\frac{C\left(\mathbf{x}^{*}\right)-C^{a}\left(\mathbf{x}^{\mathbf{a} *}\right)}{C\left(\mathbf{x}^{*}\right)}$, is shown. According to this figure, under the chosen parameter setting, for very low values of $p$ the relative cost difference can be as high as $17 \%$, while it is around $3 \%$ for low $p$ values. We would like to note that obtaining analytical expressions for the relative cost difference under the optimal solutions is not possible even for this special case with exponential leadtimes. The challenge stems from the fact that the optimality equations under our cost accounting scheme are not decoupled.

\subsection{Negative Planned Leadtimes}

As stated in Proposition 1, the planned leadtimes in our optimal solution are always non-negative. However, this is not case for the optimal solution by Atan et al. (2016). In this section, we show that $\mathbf{x}^{\mathbf{a} *}$ can have negative components. We consider a two-stage serial system with exponentially distributed leadtimes with rate $\lambda_{i}, i=0,1$. We set $\lambda_{1}=1$ and solve multiple instances by varying $\lambda_{0}$, and hence $\mathbb{E}\left[T_{0}\right]=\lambda_{0}^{-1}$. In all instances $h_{0}=h_{1}=1$ and the desired service level $\alpha=90 \%$. In Figure 4, we plot $x_{1}^{*}$ and $x_{1}^{* a}$ as function of $\mathbb{E}\left[T_{0}\right]$.

When the average leadtime of stage 0 gets much longer than the average leadtime of stage 1 , the planned lead time $x_{1}^{a *}$ decreases and becomes negative. On the other hand, $x_{1}^{*}$ stays positive and close to $\mathbb{E}\left[T_{1}\right]$. Hence, when $\mathbb{E}\left[T_{0}\right]>\mathbb{E}\left[T_{1}\right]$, our cost function leads to a meaningful optimal solution. When $\mathbb{E}\left[T_{1}\right]>\mathbb{E}\left[T_{0}\right]$, both cost functions lead to a similar optimal solution.

The same behavior can be observed for symmetric assembly systems (equal holding costs and 


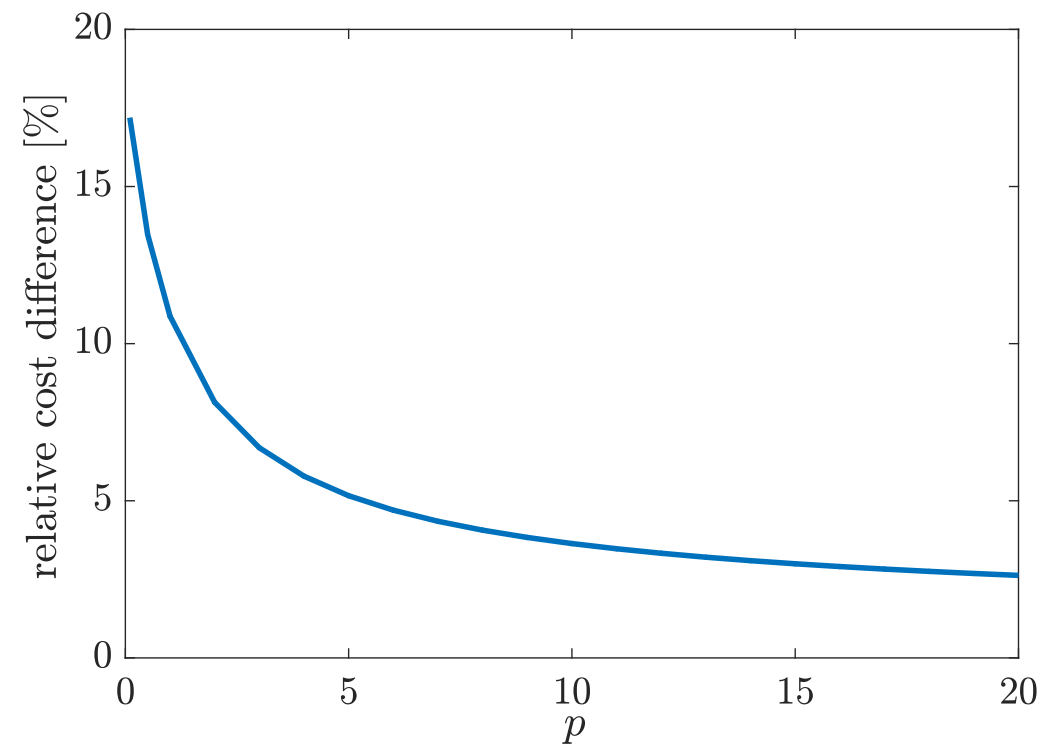

Figure 3: Relative cost difference between the two cost functions.

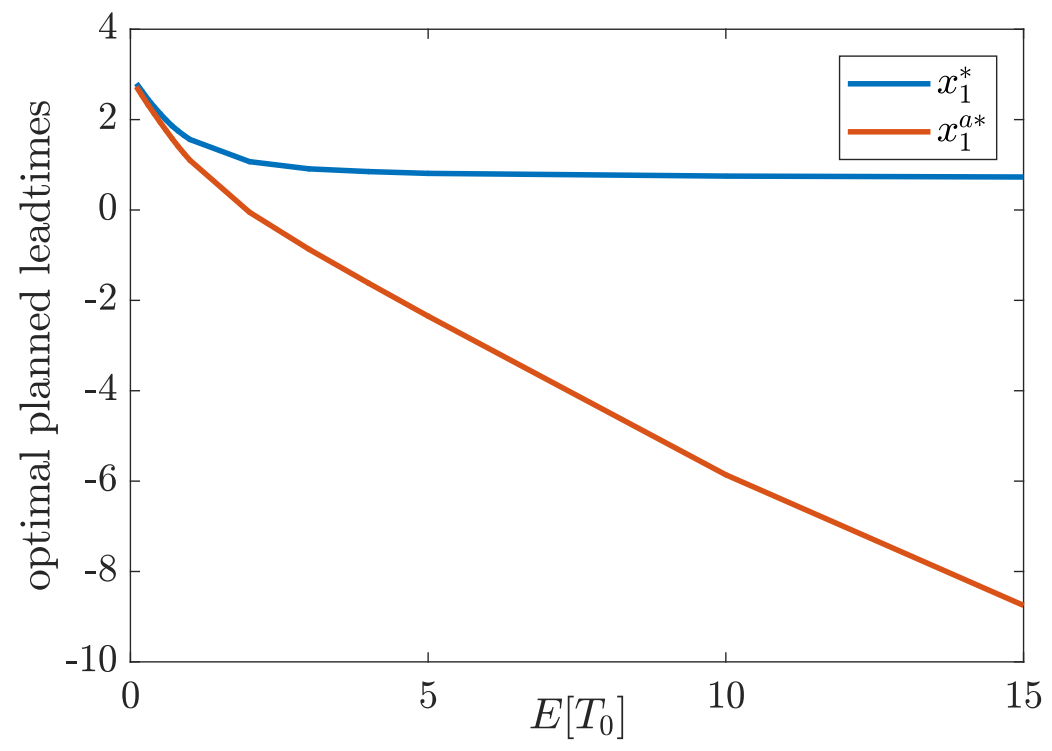

Figure 4: Optimal planned leadtimes for stage 1 for different values of $\lambda_{0}$ 
identical distributions for all subassemblies) by comparing $\max _{i \in V}\left\{T_{i}\right\}$ to $T_{0}$. For asymmetric assembly systems, a negative optimal planned leadtime is typically expected for a subassembly stage $i$ with $\mathbb{E}\left[T_{0}\right]>>\mathbb{E}\left[T_{i}\right]$.

\section{Concluding Remarks}

In this paper we study the problem of setting planned leadtimes in a configure-to-order assembly system with random leadtimes. We introduce a new cost function, which besides holding costs and a penalty cost for late delivery, also accounts for not meeting intermediate deadlines. The objective is to determine the optimal planned leadtimes, which minimize the total expected cost.

Our methodology relies on introducing a blame policy. Given that a product is delivered late, this policy identifies which stage should be blamed for the lateness. We show that for a system that is planned according to the optimal planned leadtime solution, the probability that a stage is blamed is proportional to the value the stage adds to the final product. We prove this statement by deriving a set of Newsvendor equations, that are only satisfied by the optimal planned leadtime solution.

The same system with a different cost function has been studied in Atan et al. (2016). We compare our optimal solution with their optimal solution and provide analytical results to point multiple structural differences. In addition, the two solutions are compared in numerical experiments. These experiments show that if the service level requirement is high, the difference between the optimal costs is marginal. However, the difference in the optimal planned leadtimes is significant. Our solution results in a significantly higher probability that intermediate deadlines are met. Also, negative optimal planned leadtimes, a problem faced when using the other cost function, are not possible for our cost function.

Future research might focus on combining the holding cost accounting schemes in this paper and the one studied in Atan et al. (2016). Hence, a model which accounts for both material and value added costs with the former charged from the planned start time and the latter charged from the actual start time. Another research direction can be on extending the framework to more general networks. Fast numerical approximations for computing optimal leadtimes in large networks should also be developed. Another future work might consider systems with dependent leadtimes. As leadtimes for different suppliers often correlate, this is a very relevant research direction. Our preliminary analysis shows that for some specific cases, our results also hold for dependent leadtimes. Future work should focus on identifying these special cases and formulating the conditions under which the assumption of independent leadtimes can be relaxed. Finally, note that we do not penalize the stages for being blamed for the lateness of the system. Another future work might be to consider a cost function such that each stage is penalized for the late delivery. Then, different from our study, 
the optimal solution would depend on the blame policy. Finding the optimal blame policy can be challenging yet very interesting and relevant extension.

\section{References}

Atan, Z., Ahmadi, T., Stegehuis, C., de Kok, T. \& Adan, I. (2017), 'Assemble-to-Order Systems: A Review', European Journal of Operational Research 261(3), 866-879.

Atan, Z., de Kok, T., Dellaert, N. P., van Boxel, R. \& Janssen, F. (2016), 'Setting Planned Leadtimes in Customer-Order-Driven Assembly Systems', Manufacturing $\&$ Service Operations Management 18(1), 122-140.

URL: http://dx.doi.org/10.1287/msom.2015.0565

Axsäter, S. (2005), 'Planning order releases for an assembly system with random operation times', OR Spectrum 27(2-3), 459-470.

Ben-Ammar, O., Dolgui, A. \& Wu, D. D. (2018), 'Planned lead times optimization for multi-level assembly systems under uncertainties', Omega 78, 39 - 56 .

URL: http://www.sciencedirect.com/science/article/pii/S0305048316310180

Buzacott, J. \& Shanthikumar, J. (1994), 'Safety stock versus safety time in MRP controlled production systems', Management Science 40(5), 1678-1689.

Chauhan, S., Dolgui, A. \& Proth, J.-M. (2009), 'A continuous model for supply planning of assembly systems with stochastic component procurement times', International Journal of Production Economics 120(2), 411-417.

de Kok, T. G. \& Fransoo, J. C. (2003), 'Planning supply chain operations: definition and comparison of planning concepts', Handbooks in operations research and management science 11, 597-675.

Elhafsi, M. (2002), 'Optimal leadtimes planning in serial production systems with earliness and tardiness costs', IIE Transactions 34(3), 233-243.

URL: $h t t p: / / d x . d o i . o r g / 10.1023 / A: 1012473114130$

Gong, L., de Kok, T. \& Ding, J. (1996), 'Optimal leadtimes planning in a serial production system', Management Science 40(5), 629-632.

Hopp, W. \& Spearman, M. (1993), 'Setting safety leadtimes for purchased components in assembly systems', IIE Transactions 25(2), 2-11. 
Matsuura, H. \& Tsubone, H. (1993), 'Setting planned lead times in capacity requirements planning', Journal of the Operational Research Society 44(8), 809-816.

Matsuura, H., Tsubone, H. \& Kanezashi, M. (1996), 'Setting planned lead times for multi-operation jobs', European Journal of Operational Research 88(2), 287-303.

Shore, H. (1995), 'Setting safey lead-times for purchased components in assembly systems: a general solution procedure', IIE Transactions 27(5), 638-645.

Song, D., Earl, C. \& Hicks, C. (2001), 'Stage due date planning for multistage assembly systems', International Journal of Production Research 39(9), 1943-1954.

Trietsch, D. (2006), 'Optimal feeding buffers for projects or batch supply chains by an exact generalization of the newsvendor result', International Journal of Production Research 44(4), 627-637.

Trietsch, D. \& Baker, K. R. (2012), ' $\{$ PERT $\}$ 21: Fitting pert/cpm for use in the 21st century', International Journal of Project Management 30(4), 490 - 502.

URL: //www.sciencedirect.com/science/article/pii/S026378631100127X

Weeks, J. (1981), 'Optimizing planned lead times and delivery dates', $21^{\text {th }}$ Annual Conference Proceedings, American Production and Inventory Control Society pp. 177-188.

Yano, C. A. (1987a), 'Setting Planned Leadtimes in Serial Production Systems with Tardiness Costs', Management Science 33(1), 95-106.

Yano, C. A. (1987b), 'Stochastic leadtimes in two-level assembly systems', IIE Transactions 19(4), 371-378.

\section{Appendix}

\section{Proof of Lemma 1}

First we show that

$$
\frac{\partial \mathbb{E}\left[L_{0}(\mathbf{x})\right]}{\partial x_{0}}=\mathbb{E}\left[\frac{\partial L_{0}(\mathbf{x})}{\partial x_{0}}\right],
$$

or equivalently, that for every sequence $h_{1}, h_{2}, \ldots$ converging to 0 ,

$$
\lim _{n \rightarrow \infty} \frac{\mathbb{E}\left[L_{0}\left(\mathbf{x}+h_{n} \mathbf{e}_{0}\right)\right]-\mathbb{E}\left[L_{0}(\mathbf{x})\right]}{h_{n}}=\mathbb{E}\left[\frac{\partial L_{0}(\mathbf{x})}{\partial x_{0}}\right],
$$


where $\mathbf{e}_{0}$ is the unit vector with 1 at position 0 . Since $L_{0}(\mathbf{x})(\omega)=\left(t_{0}+\max _{i \in V}\left(t_{i}-x_{i}\right)^{+}-x_{0}\right)^{+}$, the derivative $\frac{\partial L_{0}(\mathbf{x})}{\partial x_{0}}(\omega)$ exists for almost all $\omega$ and

$$
\frac{\partial L_{0}(\mathbf{x})}{\partial x_{0}}(\omega)=\left\{\begin{array}{rc}
-1, & t_{0}+\max _{i \in V}\left(t_{i}-x_{i}\right)^{+}-x_{0}>0 \\
0, & t_{0}+\max _{i \in V}\left(t_{i}-x_{i}\right)^{+}-x_{0}<0
\end{array}\right.
$$

For all $\omega$,

$$
\left|\frac{L_{0}\left(\mathbf{x}+h_{n} \mathbf{e}_{0}\right)(\omega)-L_{0}(\mathbf{x})(\omega)}{h_{n}}\right| \leq 1,
$$

so by bounded convergence we can conclude that $(9)$ holds. Hence,

$$
\frac{\partial \mathbb{E}\left[L_{0}(\mathbf{x})\right]}{\partial x_{0}}=\mathbb{E}\left[\frac{\partial L_{0}(\mathbf{x})}{\partial x_{0}}\right]=-P\left(L_{0}>0\right)=-P\left(\bigcup_{i=0}^{N} A_{i}\right)=-\sum_{i=0}^{N} P\left(A_{i}\right) .
$$

This proves the lemma for the derivative of $\mathbb{E}\left[L_{0}\right]$ with respect to $x_{0}$. The proof of the derivatives with respect to $x_{i}$ for $i \in V$ proceeds along the same lines.

\section{Proof of Theorem 2}

We consider a planned leadtime vector $\mathbf{x}$. If $x_{i}<0$ for some $i \in V$, we can write $L_{0}$ as

$$
L_{0}=\left(T_{0}+\max _{j \in V}\left\{\left(T_{j}-x_{j}\right)^{+}\right\}-x_{0}\right)^{+}=\left(T_{0}+\max _{j \in V}\left\{\left(T_{j}-x_{j}+x_{i}\right)^{+}\right\}-x_{0}-x_{i}\right)^{+}
$$

and $\mathrm{x}^{\prime}$ can be constructed as follows:

$$
\begin{aligned}
x_{i}^{\prime} & =0, \\
x_{j}^{\prime} & =x_{j}-x_{i}, \quad j \in V, j \neq i, \\
x_{0}^{\prime} & =x_{0}+x_{i} .
\end{aligned}
$$

From 100 it follows that $L_{0}^{\prime}=L_{0}$ and thus $C\left(\mathbf{x}^{\prime}\right)=C(\mathbf{x})+\left(h_{0}+h_{i}^{c}\right) x_{i}<C(\mathbf{x})$. Note that $x_{j}^{\prime}>x_{j}$ for all $j \in V$. So we can successively apply this construction to the negative subassembly planned leadtimes (as long as there are any), resulting in a vector $\mathbf{x}$ with lower costs than the original one and $x_{i} \geq 0$ for all $i \in V$. Finally, if $x_{0}<0$, we can write $L_{0}$ as

$$
L_{0}=\left(T_{0}+\max _{i \in V}\left\{L_{i}\right\}-x_{0}\right)^{+}=T_{0}+\max _{i \in V}\left\{L_{i}\right\}-x_{0}
$$

and construct a vector $\mathbf{x}^{\prime}$ as follows:

$$
\begin{aligned}
x_{0}^{\prime} & =0, \\
x_{i}^{\prime} & =x_{i}, \quad i \in V .
\end{aligned}
$$

The lateness $L_{0}^{\prime}$ for $\mathbf{x}^{\prime}$ is equal to $L_{0}+x_{0}$, so $C\left(\mathbf{x}^{\prime}\right)=C(\mathbf{x})+p x_{0}<C(\mathbf{x})$. 


\section{Proof of Lemma 2}

For a subassembly stage $i \in V,(7)$ can be written as:

$$
P\left(A_{i}\right)=P\left(T_{i}-x_{i}>0, T_{i}-x_{i}>\max _{j \in V \backslash\{i\}}\left\{T_{j}-x_{j}\right\}, T_{i}-x_{i}+T_{0}-x_{0}>0\right) .
$$

If $x_{i}$ increases, each of the three events in the right-hand side decreases and thus $P\left(A_{i}\right)$ decreases as well. Only the third event depends on $x_{0}$. This event decreases if $x_{0}$ increases. The second event is the only one depending on $x_{j}$ and it increases if $x_{j}$ increases. Hence, we can conclude that $P\left(A_{i}\right)$ decreases in $x_{i}$ and $x_{0}$ and increases in any $x_{j}, j \in V \backslash\{i\}$.

\section{Proof of Lemma 3}

For the final assembly stage, we have:

$$
P\left(A_{0}\right)=P\left(\max _{i \in V}\left\{T_{i}-x_{i}\right\} \leq 0, T_{0}-x_{0}>0\right) .
$$

The first event in the right-hand side increases in each $x_{i}$ and the second event does not depend on $x_{i}$. Therefore $P\left(A_{0}\right)$ increases in each $x_{i}$. Only the second event depends on $x_{0}$ and it decreases if $x_{0}$ increases.

\section{Proof of Theorem 3}

Theorem 1 and Proposition 1 imply that there exists a non-negative solution to the Newsvendor equations of Theorem 1. To prove uniqueness we consider two non-negative vectors $\mathbf{y}$ and $\mathbf{z}$ with $y_{0} \leq z_{0}$ and show that the blame probabilities corresponding to $\mathbf{y}$ and $\mathbf{z}$ are not identical unless $\mathbf{y}=\mathbf{z}$. We define the set $U$ as

$$
U=\left\{i: i \in V, z_{i}>y_{i}\right\}
$$

For vector $\mathbf{y}$, the probability that a stage in $U$ is blamed is equal to (cf. (11)

$$
\sum_{i \in U} P\left(A_{i}\right)=P\left(\max _{i \in U}\left\{T_{i}-y_{i}\right\}>0, \max _{i \in U}\left\{T_{i}-y_{i}\right\}>\max _{j \in V \backslash U}\left\{T_{j}-y_{j}\right\}, \max _{i \in U}\left\{T_{i}-x_{i}\right\}+T_{0}-y_{0}>0\right) .
$$

Note that $(i)$ if $y_{i}$ for $i \in U$ increases, then all three events in the right-hand side decrease, (ii) if $y_{j}$ for $j \in V \backslash U$ decreases, then only the second event decreases, and (iii) if $y_{0}$ increases, only the third event decreases. Hence, if $U$ is not empty, blame probability 12 decreases if $\mathbf{y}$ is replaced by $\mathbf{z}$. If $U$ is empty, we consider

$$
P\left(A_{0}\right)=P\left(\max _{j \in V}\left\{T_{j}-y_{j}\right\} \leq 0, T_{0}-y_{0}>0\right) .
$$

By Lemma 3 we conclude that $P\left(A_{0}\right)$ decreases if $\mathbf{y}$ is replaced by $\mathbf{z}$. 


\section{Proof of Proposition 2}

Since $h_{0}>0$ and $L_{0} \geq 0$ it follows that for any $\mathbf{x}$

$$
C(\mathbf{x})=C^{a}(\mathbf{x})+h_{0} \mathbb{E}\left[L_{0}\right] \geq C^{a}(\mathbf{x}) \geq C^{a}\left(\mathbf{x}^{\mathbf{a} *}\right) .
$$

In particular this inequality is valid for $\mathbf{x}=\mathbf{x}^{*}$.

\section{Proof of Proposition 3}

For cost function $C^{a}(\cdot)$ the optimal planned leadtime $x_{0}^{a *}$ satisfies the Newsvendor equation

$$
P\left(T_{0}>x_{0}^{a *}\right)=\frac{h_{0}}{h_{0}^{c}+p}
$$

and for cost function $C(\cdot)$ the optimal planned leadtime $x_{0}^{*}$ satisfies

$$
P\left(A_{0}\right)=P\left(T_{0}>x_{0}^{*}, \max _{i \in V}\left\{L_{i}\right\}=0\right)=P\left(T_{0}>x_{0}^{*}\right) P\left(\max _{i \in V}\left\{L_{i}\right\}=0\right)=\frac{h_{0}}{h_{0}^{c}+p} .
$$

Since $P\left(\max _{i \in V}\left\{L_{i}\right\}=0\right)<1$, it follows that $x_{0}^{*}<x_{0}^{a *}$. Similar as in the proof of Theorem 3 , we define the set $U$ as

$$
U=\left\{i: i \in V, x_{i}^{a *} \geq x_{i}^{*}\right\} .
$$

If $U$ is not empty, then it holds for $x^{*}$ that the blame probability of a stage in $U$ is equal to

$$
\begin{aligned}
\sum_{i \in U} \frac{h_{i}}{h_{0}^{c}+p} & =P\left(\max _{i \in U}\left\{T_{i}-x_{i}^{*}\right\}>\max _{j \in V \backslash U}\left\{\left(T_{j}-x_{j}^{*}\right)^{+}\right\}, \max _{i \in U}\left\{T_{i}-x_{i}^{*}\right\}+T_{0}-x_{0}^{*}>0\right) \\
& \geq P\left(\max _{i \in U}\left\{T_{i}-x_{i}^{a *}\right\}>\max _{j \in V \backslash U}\left\{\left(T_{j}-x_{j}^{a *}\right)^{+}\right\}, \max _{i \in U}\left\{T_{i}-x_{i}^{a *}\right\}+T_{0}-x_{0}^{*}>0\right) \\
& >P\left(\max _{i \in U}\left\{T_{i}-x_{i}^{a *}\right\}>\max _{j \in V \backslash U}\left\{\left(T_{j}-x_{j}^{a *}\right)^{+}\right\}, T_{0}<x_{0}^{a *}, \max _{i \in U}\left\{T_{i}-x_{i}^{a *}\right\}+T_{0}-x_{0}^{*}>0\right) .
\end{aligned}
$$

The last sum at the right-hand is the blame probability of a stage in $U$ for $x^{a *}$. It should not be less but equal to the left-hand side. Hence, we conclude that $U$ is empty and thus $x_{i}^{a *}<x_{i}^{*}$ for all $i \in V$. This completes the proof of part (a). Part $b$ is also proved by contradiction. Let us assume that

$$
x_{i}^{*}+x_{0}^{*}<x_{i}^{a *}+x_{0}^{a *}, \quad i \in V .
$$

The probability of a positive lateness $L_{0}^{*}$ under $\mathbf{x}^{*}$ is equal to the Newsvendor fractile,

$$
\begin{aligned}
\frac{h_{0}^{c}}{h_{0}^{c}+p}=P\left(L_{0}^{*}>0\right) & =P\left(\max _{i \in V}\left\{\left(T_{i}-x_{i}^{*}\right)^{+}\right\}+T_{0}-x_{0}^{*}>0\right) \\
& =P\left(\max _{i \in V}\left\{\left(T_{i}-x_{i}^{a *}-x_{i}^{*}+x_{i}^{a *}\right)^{+}\right\}+T_{0}-x_{0}^{*}>0\right) \\
& \left.\geq P\left(\max _{i \in V}\left\{\left(T_{i}-x_{i}^{a *}\right)^{+}-x_{i}^{*}+x_{i}^{a *}\right)\right\}+T_{0}-x_{0}^{*}>0\right) \\
& \geq P\left(\max _{i \in V}\left\{\left(T_{i}-x_{i}^{a *}\right)^{+}\right\}-\max _{i \in V}\left\{x_{i}^{*}-x_{i}^{a *}\right\}+T_{0}-x_{0}^{*}>0\right) \\
& >P\left(\max _{i \in V}\left\{\left(T_{i}-x_{i}^{a *}\right)^{+}\right\}-x_{0}^{a *}+x_{0}^{*}+T_{0}-x_{0}^{*}>0\right) \\
& =P\left(\max _{i \in V}\left\{\left(T_{i}-x_{i}^{a *}\right)^{+}\right\}+T_{0}-x_{0}^{a *}>0\right)=P\left(L_{0}^{a *}>0\right),
\end{aligned}
$$


where we used $(b-a)^{+} \geq b^{+}-a$ for numbers $a>0$ and $b$ in the first inequality and assumption (14) in the third. This is a contradiction, since $P\left(L_{0}^{a *}>0\right)$ should also be equal to the Newsvendor fractile at the left-hand side. Hence, (14) is not valid, so there is a stage $i$ for which $x_{i}^{*}+x_{0}^{*} \geq x_{i}^{a *}+x_{0}^{a *}$.

\section{Proof of Proposition 4}

To derive the limiting behavior of $x_{0}^{a *}$, we rewrite (13) as:

$$
P\left(T_{0} \leq x_{0}^{a *}\right)=1-\frac{h_{0}}{h_{0}^{c}+p}=\alpha+\frac{h_{1}}{h_{0}^{c}+p},
$$

so

$$
x_{0}^{a *}=F_{0}^{-1}\left(\alpha+\frac{h_{1}}{h_{0}^{c}+p}\right) .
$$

Since $\frac{h_{1}}{h_{0}^{c}+p} \rightarrow 0$ as $h_{0} \rightarrow \infty$, it follows from the continuity of $F_{0}^{-1}(\cdot)$ that

$$
\lim _{h_{0} \rightarrow \infty}\left\{x_{0}^{a *}\right\}=F_{0}^{-1}(\alpha) .
$$

The planned leadtime $x_{1}^{a *}$ satisfies the Newsvendor equation

$$
P\left(T_{1}>x_{1}^{a *}, T_{0}<x_{0}^{a *}, T_{1}+T_{0}>x_{1}^{a *}+x_{0}^{a *}\right)=\frac{h_{1}}{h_{0}^{c}+p} .
$$

The right-hand side converges to 0 as $h_{0} \rightarrow \infty$. Hence the blame probability at the left-hand side also converges to 0 . Since $x_{0}^{a *}$ converges to a proper limit, this implies that $x_{1}^{a *}$ has to grow to infinity. From Proposition 3 it follows that $x_{1}^{*}>x_{1}^{a *}$, so also $x_{1}^{*}$ tends to infinity. Finally, $x_{0}^{*}$ satisfies (6), which can be rewritten to

$$
P\left(T_{1} \leq x_{1}^{*}\right) P\left(T_{0}>x_{0}^{*}\right)=\frac{h_{0}}{h_{0}^{c}+p}=1-\alpha-\frac{h_{1}}{h_{0}^{c}+p} .
$$

Since $x_{1}^{*} \rightarrow \infty$, and thus $P\left(T_{1} \leq x_{1}^{*}\right) \rightarrow 1$ as $h_{0} \rightarrow \infty$, it follows from the continuity of $F_{0}^{-1}(\cdot)$ that

$$
\lim _{h_{0} \rightarrow \infty}\left\{x_{0}^{a *}\right\}=F_{0}^{-1}(\alpha)
$$

\section{Proof of Proposition 5}

To prove the first equation of Proposition 5, we start from (6). For stage 1, this equation can be written as

$$
\begin{array}{r}
P\left(T_{0}+T_{1}>x_{1}^{*}+x_{0}^{*}, T_{1}>x_{1}^{*}\right)=\frac{h_{1}}{h_{0}^{c}+p} \\
P\left(T_{0}+T_{1}^{\prime}>x_{0}^{*} \mid T_{1}>x_{1}^{*}\right) P\left(T_{1}>x_{1}^{*}\right)=\frac{h_{1}}{h_{0}^{c}+p} .
\end{array}
$$


In the second expression, $T_{1}^{\prime}$ denotes the leadtime of stage 1 , given that it exceeds $x_{1}^{*}$. Due to the memoryless property, this random variable is again exponentially distributed with parameter $\lambda$. The sum of 2 independent exponential variables with rate $\lambda$ is an Erlang-2 distribution and thus we find the following expressions hold

$$
\begin{aligned}
P\left(T_{0}+T_{1}^{\prime}>x_{0}^{*} \mid T_{1}>x_{1}^{*}\right) & =\left(1+\lambda x_{0}^{*}\right) e^{-\lambda x_{0}^{*}} \\
P\left(T_{1}>x_{1}^{*}\right) & =e^{-\lambda x_{1}^{*}} .
\end{aligned}
$$

Hence, we obtain

$$
P\left(T_{0}+T_{1}>x_{1}^{*}+x_{0}^{*}, T_{1}>x_{1}^{*}\right)=\left(1+\lambda x_{0}^{*}\right) e^{-\lambda\left(x_{0}^{*}+x_{1}^{*}\right)},
$$

which leads to

$$
\left(1+\lambda x_{0}^{*}\right) e^{-\lambda\left(x_{0}^{*}+x_{1}^{*}\right)}=\frac{h_{1}}{h_{0}^{c}+p} .
$$

To prove the second equation of Proposition 5 we start from (6). For stage 0, this equation can be written as

$$
P\left(A_{0}\right)=P\left(T_{1}<x_{1}^{*}\right) P\left(T_{0}>x_{0}^{*}\right)=\frac{h_{0}}{h_{0}^{c}+p} .
$$

Assuming exponential distributions leads to

$$
\left(1-e^{-\lambda x_{1}^{*}}\right) e^{-\lambda x_{0}^{*}}=e^{-\lambda x_{0}^{*}}-e^{-\lambda\left(x_{1}^{*}+x_{0}^{*}\right)}=\frac{h_{0}}{h_{0}^{c}+p} .
$$

\section{Proof of Proposition 6}

In Atan et al. (2016), it is proven that

$$
P\left(T_{1}>x_{1}^{a *}, T_{0}<x_{0}^{a *}, T_{1}+T_{0}>x_{1}^{a *}+x_{0}^{a *}\right)=\frac{h_{1}}{h_{0}^{c}+p} .
$$

Assuming identically exponentially distributed leadtimes, this equation can be rewritten as

$$
\begin{aligned}
\int_{x_{1}^{a *}}^{\infty} \int_{\left(x_{1}^{a *}+x_{0}^{a *}-t_{1}\right)^{+}}^{x_{0}^{a *}} \lambda e^{-\lambda t_{1}} \lambda e^{-\lambda t_{0}} d t_{0} d t_{1} & \\
& =\int_{x_{1}^{a *}}^{x_{1}^{a *}+x_{0}^{a *}} \int_{x_{1}^{a *}+x_{0}^{a *}-t_{1}}^{x_{0}^{a *}} \lambda^{2} e^{-\lambda\left(t_{1}+t_{0}\right)} d t_{0} d t_{1}+\int_{x_{1}^{a *}+x_{0}^{a *}}^{\infty} \int_{0}^{x_{0}^{a *}} \lambda^{2} e^{-\lambda\left(t_{1}+t_{0}\right)} d t_{0} d t_{1} .
\end{aligned}
$$


The first term of the right-hand side is computed as follows:

$$
\begin{aligned}
\int_{x_{1}^{a *}}^{x_{1}^{a *}+x_{0}^{a *}} \int_{x_{1}^{a *}+x_{0}^{a *}-t_{1}}^{x_{0}^{a *}} \lambda^{2} e^{-\lambda\left(t_{1}+t_{0}\right)} d t_{1} d t_{0} & =\lambda \int_{x_{1}^{a *}}^{x_{1}^{a *}+x_{0}^{a *}}\left[-e^{-\lambda\left(t_{1}+t_{0}\right)}\right]_{x_{1}^{a *}+x_{0}^{a *}-t_{1}}^{x_{0}^{a *}} d t_{1} \\
& =\lambda \int_{x_{1}^{a *}}^{x_{1}^{a *}+x_{0}^{a *}} e^{-\lambda\left(x_{1}^{a *}+x_{0}^{a *}\right)} d t_{1}-\lambda \int_{x_{1}^{a *}}^{x_{1}^{a *}+x_{0}^{a *}} e^{-\lambda\left(t_{1}+x_{0}^{a *}\right)} d t_{1} \\
& =\lambda x_{0}^{a *} e^{-\lambda\left(x_{1}^{a *}+x_{0}^{a *}\right)}+e^{-\lambda\left(x_{1}^{a *}+x_{0}^{a *}+x_{0}^{a *}\right)}-e^{-\lambda\left(x_{1}^{a *}+x_{0}^{a *}\right) .}
\end{aligned}
$$

The second term of the right-hand side of $(15)$ can be rewritten as

$$
\begin{aligned}
\int_{x_{1}^{a *}+x_{0}^{a *}}^{\infty} \int_{0}^{x_{0}^{a *}} \lambda^{2} e^{-\lambda\left(t_{1}+t_{0}\right)} d t_{0} d t_{1} & =P\left(T_{1}>x_{1}^{a *}+x_{0}^{a *}\right) P\left(T_{0}<x_{0}^{a *}\right) \\
& =e^{-\lambda\left(x_{1}^{a *}+x_{0}^{a *}\right)}\left(1-e^{\left.-\lambda x_{0}^{a *}\right)}\right. \\
& =e^{-\lambda\left(x_{1}^{a *}+x_{0}^{a *}\right)}-e^{-\lambda\left(x_{1}^{a *}+x_{0}^{a *}+x_{0}^{a *}\right)} .
\end{aligned}
$$

Finally, inserting these results into (15) leads to $\lambda x_{0}^{a *} e^{-\lambda\left(x_{1}^{a *}+x_{0}^{a *}\right)}=\frac{h_{1}}{h_{0}^{c}+p}$.

In Atan et al. (2016) it is also proven that

$$
P\left(T_{0}>x_{0}^{a *}\right)=\frac{h_{0}}{h_{0}^{c}+p} .
$$

By inserting the inverse cumulative probability for an exponential distribution, which is $e^{-\lambda x_{0}^{a *}}$ it directly follows that

$$
e^{-\lambda x_{0}^{a *}}=\frac{h_{0}}{h_{0}^{c}+p}
$$

\section{Proof of Proposition 7}

For this proof we use the optimality equations for stage 1 from Propositions 5 and 6 and rewrite the terms:

$$
\begin{aligned}
\left(1+\lambda x_{0}^{*}\right) e^{-\lambda\left(x_{0}^{*}+x_{1}^{*}\right)} & =\lambda x_{0}^{a *} e^{-\lambda\left(x_{1}^{a *}+x_{0}^{a *}\right)} \\
e^{-\lambda\left(x_{0}^{*}+x_{1}^{*}-x_{1}^{a *}-x_{0}^{a *}\right)} & =\frac{\lambda x_{0}^{a *}}{1+\lambda x_{0}^{*}} \\
x_{1}^{*}-x_{1}^{a *} & =-\frac{1}{\lambda} \ln \left(\frac{\lambda x_{0}^{a *}}{1+\lambda x_{0}^{*}}\right)+x_{0}^{a *}-x_{0}^{*}
\end{aligned}
$$

From Proposition 4 it follows that $\lim _{h_{0} \rightarrow \infty}\left\{x_{0}^{*}-x_{0}^{a *}\right\}=0$ and thus we find

$$
\lim _{h_{0} \rightarrow \infty}\left\{x_{1}^{*}-x_{1}^{a *}\right\}=-\frac{1}{\lambda} \ln \left(\frac{\lambda x_{0}^{*}}{1+\lambda x_{0}^{*}}\right) .
$$

